\title{
Postpneumonectomy syndrome related to the thickness of the fat tissue in the anterior mediastinum: a retrospective observational study
}

\author{
Yasoo Sugiura, Takashi Nakayama, Toshinori Hashizume \\ Department of General Thoracic Surgery, National Hospital Organization, Kanagawa National Hospital, Kanagawa Japan \\ Contributions: (I) Conception and design: Y Sugiura; (II) Administrative support: T Hashizume; (III) Provision of study materials or patients: All \\ authors; (IV) Collection and assembly of data: Y Sugiura; (V) Data analysis and interpretation: Y Sugiura; (VI) Manuscript writing: All authors; (VII) \\ Final approval of manuscript: All authors. \\ Correspondence to: Yasoo Sugiura. Department of General Thoracic Surgery, National Hospital Organization Kanagawa National Hospital, 666-1 \\ Ochiai Hadano, Kanagawa 257-8585, Japan. Email: dryasoo@outlook.com.
}

Background: Postpneumonectomy syndrome (PPS) is a life-threatening condition characterized by the extrinsic compression of the mainstem bronchus after pneumonectomy, causing rapidly progressive dyspnea. Information regarding the predictive factors of this condition is limited. Thus, the current study aimed to identify the predictive findings that can help prepare the treatment of PPS in advance.

Methods: The present study is a retrospective observational study. We reviewed the medical records of 12 consecutive patients who underwent pneumonectomy for lung cancer or tuberculosis between 2009 and 2020. The anatomical findings evaluated via computed tomography scan, nutritional status assessed using laboratory data, respiratory function, intraoperative variables between PPS and non-postpneumonectomysyndrome patients were compared.

Results: There were two female patients who presented with left PPS (PPS 1 and PPS 2 aged 73 and 55 years, respectively). The median age of 10 non-postpneumonectomy-syndrome patients ( $\mathrm{n}=6$, men and $\mathrm{n}=4$ women) was 66 (range, 54-76 years). Four and six patients underwent right and left pneumonectomy, respectively. The fat tissue thickness in the anterior mediastinum was significantly thinner in PPS than in non-postpneumonectomy-syndrome patients. However, the body mass index (BMI) and other nutritional parameters did not differ between PPS and non-postpneumonectomy-syndrome patients. The median vital capacity was higher in PPS than in non-postpneumonectomy-syndrome patients. The decrement of respiratory function and body weight after pneumonectomy did not differ between two groups. The intraoperative variables did not also differ between two groups.

Conclusions: The fat tissue in the anterior mediastinum between the bilateral lungs is an anchor that can help maintain the normal position of the mediastinum after pneumonectomy. Thus, the fat tissue thickness between the bilateral lungs can be a predictive factor for the occurrence of PPS before surgery.

Keywords: Pneumonectomy; postpneumonectomy syndrome (PPS); lung cancer; mediastinum

Submitted Jul 05, 2020. Accepted for publication Sep 23, 2020.

doi: $10.21037 /$ jtd-20-2370

View this article at: http://dx.doi.org/10.21037/jtd-20-2370

\section{Introduction}

Postpneumonectomy syndrome (PPS) is a rare lifethreatening condition characterized by extrinsic compression of the trachea and mainstem bronchus after pneumonectomy.
Several mechanisms associated with the condition have been reported $(1,2)$. First, an excessive shift of the heart and mediastinum toward the side of the pneumonectomy is observed. Second, there is a significant rotation of the great 
vessels. Third, hyperinflation of the remaining lung occurs. The trachea and/or main bronchus are compressed against the vertebral column or aorta. Consequently, patients develop progressive dyspnea, inspiratory stridor, and recurrent pneumonia at least 6 months after surgery and the condition can be fatal if left untreated.

There are several treatments for PPS. That is, the position of the shifted mediastinum can be treated with surgery, intrapleural gas injection therapy, or intrathoracic tissue expander (3-5). Another option is the placement of expandable stents at the compressed portion of the airway $(6,7)$.

PPS causes severe asphyxia and even death. In this study, two adult patients died of this condition, which rapidly developed to progressive dyspnea. To identify predictive factors that can help prepare the treatment of PPS in advance, we assessed the data of 12 adult patients who underwent pneumonectomy, including two who presented with PPS in our institution.

We present the following article in accordance with the STROBE reporting checklist (available at http://dx.doi. org/10.21037/jtd-20-2370).

\section{Methods}

\section{Patients}

The present study is a retrospective observational study. The study was conducted in accordance with the Declaration of Helsinki (as revised in 2013). The Committee for Protection of Human Subjects, the local institutional review board of National Hospital Organization, Kanagawa National Hospital approved this study (The approval number of Institutional Review Board of Kanagawa Hospital: R2-3). Because of the retrospective nature of the study, patient informed consent or ethical statement was not required. All patients who underwent pneumonectomy for lung cancer or tuberculosis at our institution between January 2009 and March 2020 were identified from a maintained surgical database of our institution. All patients or families were contacted, and they agree to provide information about their clinical courses.

\section{Data collection}

We analyzed the medical records from three viewpoints.
First, we assessed some mediastinum structures using computed tomography (CT) scan images. Second, the respiratory function of the patients before surgery were compared. Third, nutritional status was assessed using laboratory information obtained before surgery. Data, such as height, body weight, blood examination results, CT images, chest radiography images, presenting signs and symptoms, surgical approach used, date of surgery, interval between pneumonectomy and the development of PPS, preoperative and postoperative radiographic and pulmonary function test results, operation time, the amount of blood loss, the presence of adhesion between thoracic wall and lung, postoperative morbidity, and mortality and last followup visit or date of death, were obtained from the medical records of the patients. The patients were followed up every three or four months after surgery by five years in principle. There was not missing patient in the observation period.

\section{Definition of parameters}

The parameters in the axial images of CT scan conducted before surgery, which may indicate the stability of the mediastinal organs, were assessed (Figure 1). The thinnest portion of the fat tissue between bilateral thoracic cavities in the anterior mediastinum at the level of the carina was referred to as $\mathrm{T}$ (Figure 1A). The length of the fat tissue between the bilateral thoracic cavities in the anterior mediastinum at the level of the carina, with a thickness $\leq 1 \mathrm{~mm}$, was referred to as $\mathrm{L}_{\mathrm{A}}$ (Figure $1 B$ ). The length between the sternum and the vertebra at the level of the carina was referred to as $\mathrm{L}_{\mathrm{B}}$ (Figure $1 C$ ). The length between the carina and the vertebra at the level of the carina was referred to as $\mathrm{L}_{\mathrm{C}}$ (Figure $1 D$ ). The area of the fat tissue in the anterior mediastinum at the level of the carina was evaluated. To assess nutritional status, the body mass index (BMI), prognostic nutritional index (PNI), neutrophil-lymphocyte ratio (NLR), and plateletlymphocyte ratio (PLR) of the patients were evaluated (8-10). BMI was defined as weight in kilograms divided by height in meters squared. $\Delta \mathrm{BW}$ was defined as the decrement of body weight in the first 6-12 months after pneumonectomy. PNI was calculated based on the serum albumin concentration and peripheral blood lymphocyte count. Based on the respiratory function test before surgery, vital capacity (VC) and forced expiratory volume in 

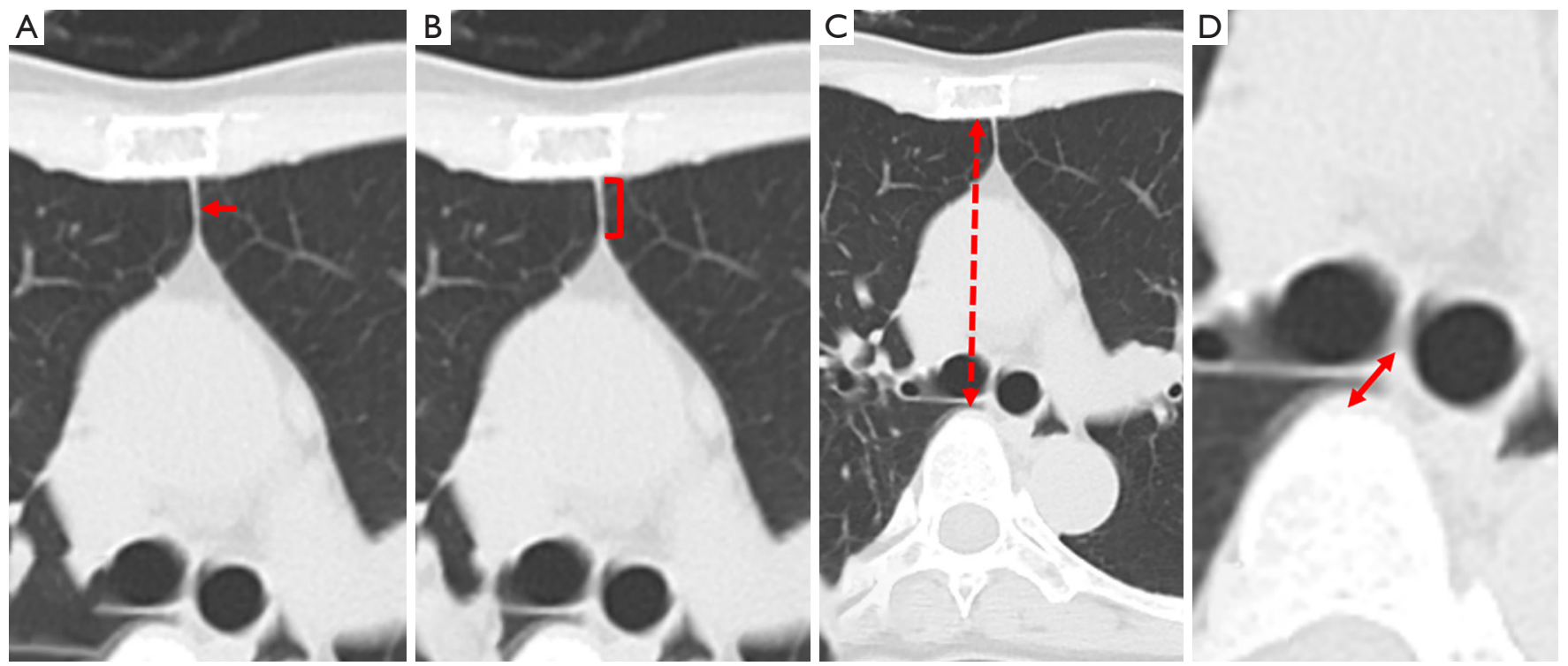

Figure 1 (A) The thinnest portion of the fat tissue between the bilateral thoracic cavities in the anterior mediastinum at the level of the carina was referred to as T. (B) The length of the fat tissue between bilateral the thoracic cavities in the anterior mediastinum at the level of the carina, with a thickness $\leq 1 \mathrm{~mm}$, was referred to as $\mathrm{L}_{\mathrm{A}}$. (C) The length between the sternum and the vertebra at the level of the carina was referred to as $L_{B}$. (D) The length between the carina and vertebra was referred to as $L_{C}$.

1 second (FEV1) were assessed. The predicted postoperative VC (ppo-VC) and predicted postoperative FEV1 (ppoFEV1) were calculated using the following formula: ppo$\mathrm{VC}$ or ppo-FEV1 = preoperative $\mathrm{VC}$ or ppo-FEV1 $\times($ No. of segments remaining/total No. of segments) (11). $\Delta \mathrm{VC}$ was defined as the decrement of VC the first 6-12 months after pneumonectomy. $\triangle \mathrm{FEV} 1$ was defined as the decrement of FEV1 the first 6-12 months after pneumonectomy.

\section{Statistical analysis}

Continuous non-normal data were expressed as medians with range (minimum and maximum). In univariate analysis for the two groups, categorical variables were compared using the $\chi^{2}$ test or Fisher's exact test, while Student $t$-test was used to compare normally distributed continuous variables, and non-normal data were tested with MannWhitney $U$ test with IBM SPSS Statistics version 25 (IBM, Armonk, NY, USA). A P value $<0.05$ was considered statistically significant.

\section{Results}

There were 12 patients undergone pneumonectomy between January 2009 and March 2020 in our institution. PPS occurred in 2 patients and did not occur in 10 patients. Of the 12 patients in this study, 6 were men and 6 were women. In total, 10 patients underwent pneumonectomy for lung cancer and 2 for tuberculosis empyema with fistula. Two patients with lung cancer received neo-adjuvant chemotherapy, and anti-tuberculosis agents (rifampicin, isoniazid, pyrazinamide, and ethambutol) were administered in two patients with tuberculosis empyema before and after surgery (Table 1).

There were two female patients who presented with PPS [PPS-1 (Figure 2A,B,C) and PPS-2 (Figure 2D,E,F)] aged 73 and 55 years, respectively. The two patients underwent left pneumonectomy, and PPS-1 and PPS-2 died 31 and 21 months after surgery, respectively. In both cases, dyspnea, which particularly occurred when they stooped down, gradually developed 6 months after surgery. They suddenly presented with sever hypoxia, which is an emergency condition. Respiratory support via intubation and bag ventilation exacerbated hypoxia and resulted in death. There was no clinical evidence to deteriorate pulmonary function other than PPS.

The median age of 10 non-PPS patients $(n=6$, men 
Table 1 Characteristics of the patients

\begin{tabular}{|c|c|c|c|c|}
\hline Characteristic & PPS-1 & PPS-2 & Non-PPS & $P$ value \\
\hline Gender (cases) & Female & Female & Female: 4 , male: 6 & 0.224 \\
\hline Affected side (cases) & Left & Left & Left: 6 , right: 4 & 0.079 \\
\hline Indication for pneumonectomy & Lung cancer & Lung cancer & Lung cancer: 8 , tuberculosis: 2 & \\
\hline Adjuvant chemotherapy (cases) & CDDP + PEM: two courses & S-1 & 4 & \\
\hline
\end{tabular}

Preoperative characteristics of the patients who underwent pneumonectomy. PPS, postpneumonectomy syndrome; CDDP, cisplatin; PEM, pemetrexed; S-1, tegafur-gimeracil-oteracil.
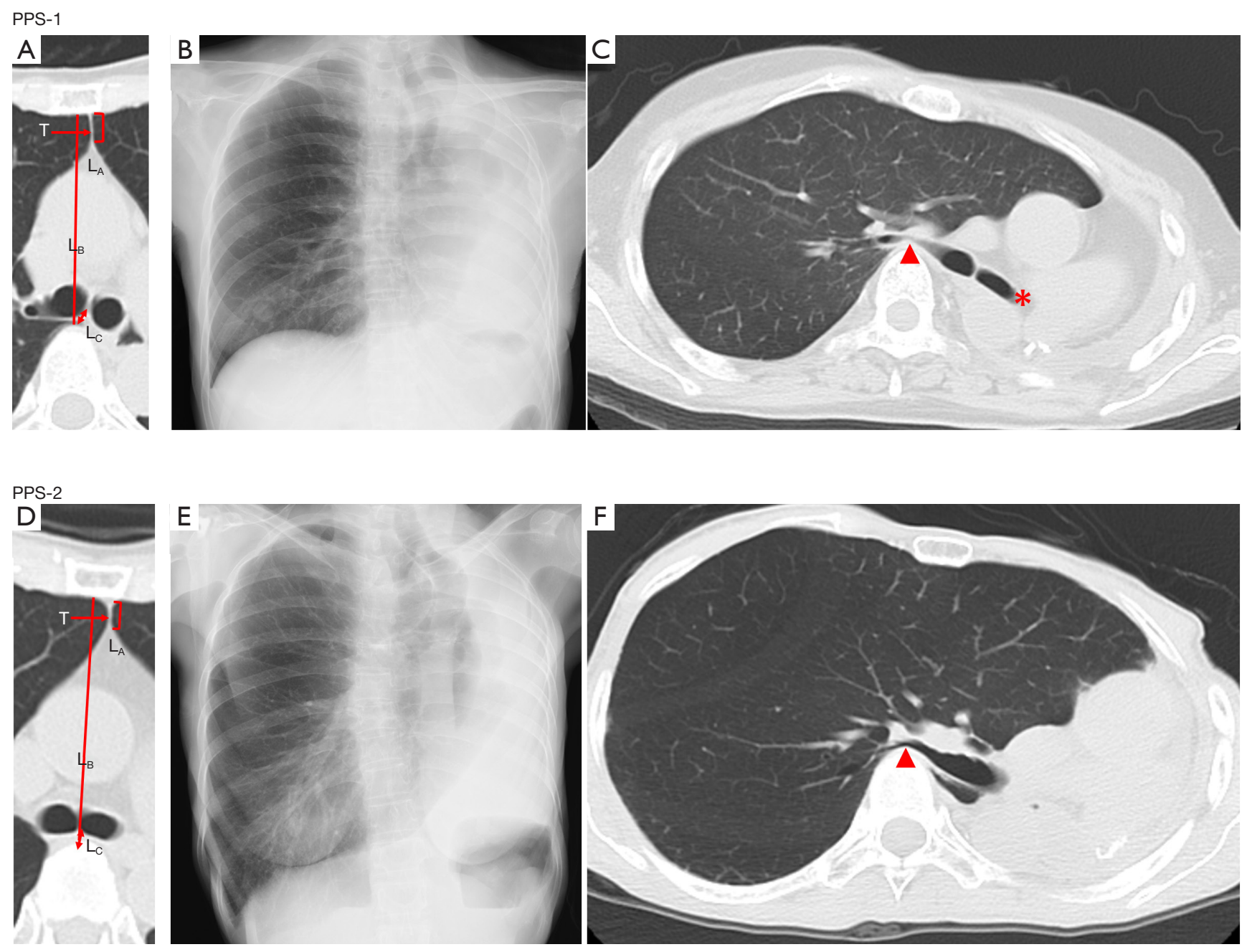

Figure $2(\mathrm{~A}, \mathrm{~B}, \mathrm{C})$ are images of PPS-1, and (D,E,F) are images of PPS-2. (A and D) are the CT scan at the level of the carina before surgery. $\mathrm{B}$ and $\mathrm{E}$ are the chest radiography image after PPS. (C and F) are the CT scan at the level of the carina after PPS. Chest radiography image (B and E) showed hyperinflation of the right lung, shift of the trachea and the mediastinum. CT scan image (C and F) showed that the right lung compressed the right main bronchus on the vertebra. The arrowhead indicates the compressed and narrowed right main bronchus. The asterisk indicates the stump of the left main bronchus. PPS, postpneumonectomy syndrome; CT, computed tomography. 
Table 2 Comparison of the CT scan findings, nutritional status, respiratory function and intraoperative variables between PPS patients and nonPPS patients

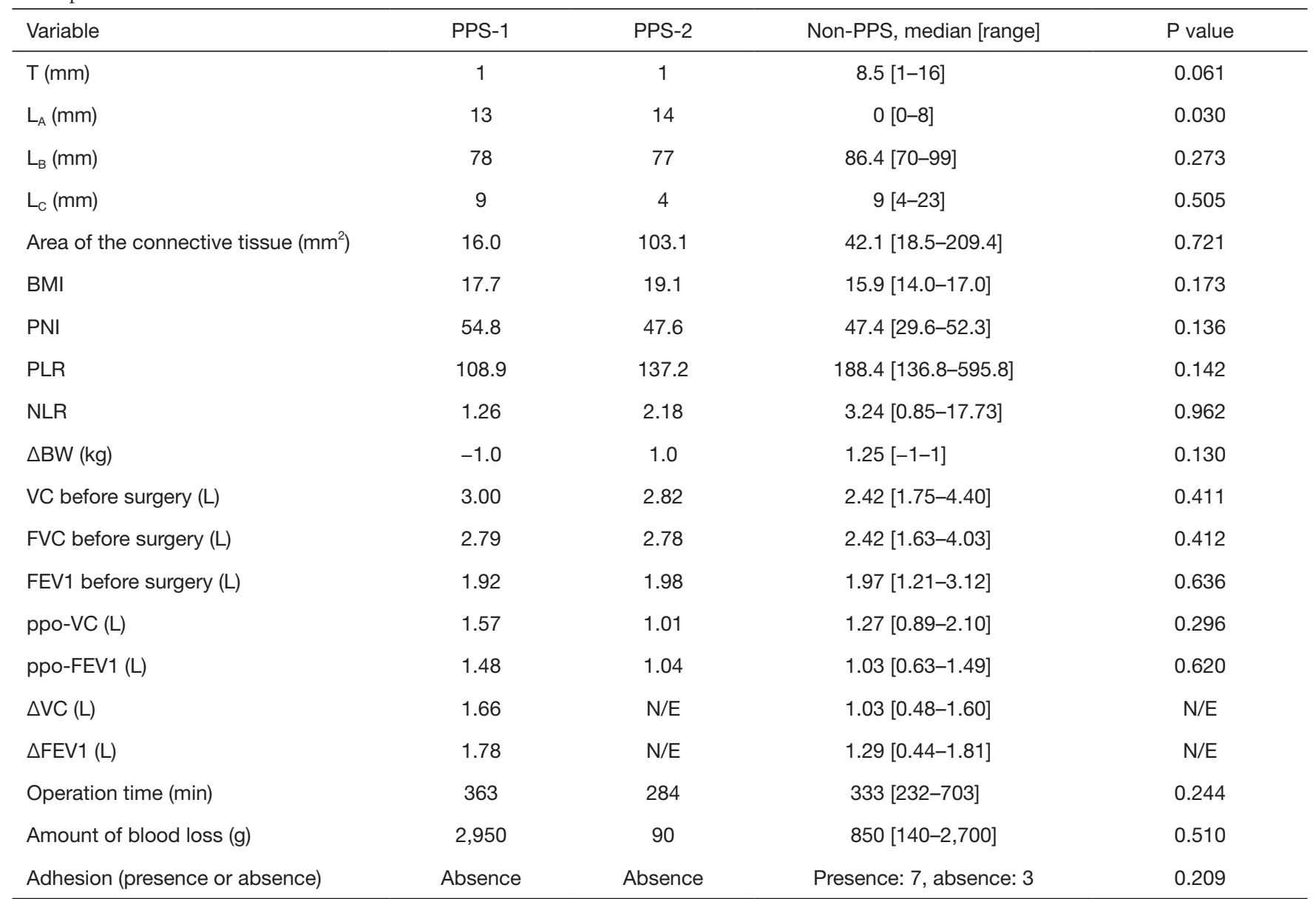

PPS, postpneumonectomy syndrome; T, thinnest fat tissue between the bilateral lungs in the anterior mediastinum; LA, length of the T, with a thickness $\leq 1 \mathrm{~mm}$; LB, length between the sternum and the vertebra at the level of the carina; LC, the length between the carina and the vertebra at the level of the carina; BMI, body mass index; PNI, prognostic nutritional index; PLR, platelet-lymphocyte ratio; NLR, neutrophil-lymphocyte ratio; $\triangle B W$, the decrement of body weight in the first 6-12 months after surgery; VC, vital capacity; FEV1, forced expiratory volume in 1 second; ppo-VC, predicted postoperative vital capacity; ppo-FEV1, predicted postoperative forced expiratory volume in 1 second; $\triangle \mathrm{VC}$, the decrement of $\mathrm{VC}$ the first 6-12 months after pneumonectomy; $\triangle \mathrm{FEV} 1$, he decrement of FEV1 the first 612 months after pneumonectomy; N/E, not examined.

and $n=4$, women) was 66 (range, 54-76) years. Both PPS1 and PPS-2 were female. In total, four and six patients underwent right and left pneumonectomy, respectively. The two groups, which are PPS patients and non-PPS patients, did not differ significantly in age, gender and affected side. The median follow-up time was 35.5 months.

The results of comparison of the CT scan findings, nutritional status, respiratory function, and intraoperative variables between PPS patients and non-PPS patients were shown in Table 2. Based on the CT scan images before surgery, the T of PPS-1, PPS-2 and the median T of non-PPS patients were 1,1 , and 8.5 (range, 1-16) mm, respectively. The $\mathrm{L}_{\mathrm{A}}$ of PPS-1, PPS-2 and the median $\mathrm{L}_{\mathrm{A}}$ of non-PPS patients were 13,14 , and 0 (range, $0-8$ ) $\mathrm{mm}$, respectively. The $\mathrm{L}_{\mathrm{B}}$ of PPS-1, PPS-2 and the median $\mathrm{L}_{\mathrm{A}}$ of non-PPS patients were 78, 77, and 86.4 (range, 70-99) mm, respectively. The $\mathrm{L}_{\mathrm{C}}$ of PPS-1, PPS2 and the median LC of non-PPS patients were 9, 9, and 9 (range, 4-23) $\mathrm{mm}$, respectively. The area of the fat tissue of PPS-1, PPS-2 and the median value of non-PPS patients were 16.0, 103.1, and 42.1 (range, 18.5-209.4) $\mathrm{mm}^{2}$, respectively. The $\mathrm{L}_{\mathrm{A}}$ significantly differed between PPS and non-PPS patients $(\mathrm{P}=0.030)$. The $\mathrm{T}$ has also a tendency to be thinner in 
PPS patients $(\mathrm{P}=0.61)$. This result indicated that the partition between the bilateral thoracic cavities was thin in PPS patients.

The BMI of PPS-1, PPS-2 and the median BMI of nonPPS patients were 17.7, 19.1, and 15.9 (range, 14.0-17.0), respectively. The PNI were 54.8, 47.6, and 47.4 (range, 29.6-52.3), respectively. The PLR were 108.9, 137.2, and 188.4 (range, 136.8-595.8), respectively. The NLR were $1.26,2.18$, and 3.24 (range, $0.85-17.73$ ), respectively. The $\triangle \mathrm{BW}$ of PPS-1, PPS-2 and the median $\triangle \mathrm{BW}$ of non-PPS patients were $-1.0,1.0$ and 1.25 (range, -1.0 to 14.0 ) $\mathrm{kg}$, respectively. The two groups did not differ significantly in BMI, PNI, PLR, NLR and $\triangle B W$. These results indicated that the nutrition status of PPS patients was not inferior to that of non-PPS patients.

The VC of PPS-1, PPS-2 and the median VC of nonPPS patients were 3.00, 2.82, and 2.42 (range, 1.75-4.40) L, respectively. The FEV1 of PPS-1, PPS-2 and the median FEV1 of non-PPS patients were 1.92, 1.98, and 1.97 (range, 1.21-3.12) L, respectively. The ppo-VC of PPS-1, PPS-2 and the median ppo-VC of non-PPS patients were $1.57,1.01$ and 1.27 (range, 0.89-2.10) L, retrospectively. The ppoFEV1 of PPS-1, PPS-2 and the median ppo-FEV1 of nonPPS patients were $1.48,1.04$, and 1.03 (range, 0.63-1.49), retrospectively. The $\triangle \mathrm{VC}$ of PPS-1, PPS-2 and the median $\Delta \mathrm{VC}$ of non-PPS patients were 1.66 , not examined, and 1.03 (0.48-1.60) L, respectively. The $\triangle \mathrm{FEV} 1$ of PPS-1, PPS-2 and the median $\triangle \mathrm{FEV} 1$ of non-PPS patients were 1.78 , not examined, and $1.29(0.44-1.81) \mathrm{L}$, respectively. The two groups did not differ significantly in VC before surgery, FEC before surgery, FEV1 before surgery, ppo-VC, ppoFEV, VC and FEV1. The VC and FVC of PPS patients was higher than the median of non-PPS patients. The ppoFEV1 of PPS patients was higher than the median of nonPPS patients. The $\triangle \mathrm{VC}$ and $\triangle \mathrm{FEV} 1$ were more than the median of non-PPS patients.

The operation time of PPS-1, PPS- 2 and the median operation time of non-PPS patients were 363,284 , and 333 (range, 232-703) minutes, respectively. The amount of blood loss of PPS-1, PPS-2 and the median amount of blood loss of non-PPS patients were 2,950, 90, and 850 (range, 140-2,700) g, respectively. In PPS-1 and PPS-2, the adhesion between thoracic wall and lung was absence. In 7 non-PPS patients, adhesion was presence. On the other hand, in 2 non-PPS patients, adhesion was absence. The two groups did not differ significantly in the operation time, the amount of blood loss and adhesion.

\section{Discussion}

Dyspnea develops rapidly after the development of PPS, and this condition is fatal if left untreated. Several treatments for this condition have been reported. One is repositioning of the mediastinum via surgery and filling of the postpneumonectomy space with a non-absorbable material (3-5). Another option is endobronchial intervention $(6,7)$. However, the treatments must be prepared before the development of PPS. Grillo et al. also mentioned that occurrence of the syndrome is unpredictable. The incidence would seem to be rare enough so that prophylactic steps that might add complexity to pneumonectomy do not appear to be justified (12). Therefore, whether PPS occurs before progressive dyspnea should be predicted and investigation for the predictive factors of the occurrence of postpneumonectomy is worth.

Mehran et al. described that risk factors for the PPS include age and sex of the patient. Elasticity of the mediastinal tissues is one of the risk factors. Women, in general, and young patients can shift more than elderly patients (1). We focused on the anatomical structures around the mediastinum, which maintain the normal position of the mediastinum after pneumonectomy.

From an anatomical viewpoint, the $\mathrm{L}_{\mathrm{A}}$ significantly differed between PPS and non-PPS patients $(\mathrm{P}=0.030)$. The $T$ has also a tendency to be thinner in PPS patients $(\mathrm{P}=0.61)$. This result indicated that the partition between the bilateral thoracic cavities in the anterior mediastinum was thin in PPS patients. The density of the tissue in the anterior mediastinum on CT scan in non-PPS patients was compatible with the fat density, with an average CT value of -83.6. However, the BMI of the two PPS patients were 17.1 and 19.1, and the median BMI of non-PPS was 15.9. This result indicated that the PPS patients were not thinner than non-PPS patients in physical constitution. Regarding to the nutritional parameters, the NLR, PLR and PNI did not significantly differ between PPS cases and non-PPS patients. In terms of BMI, NLR, PLR and PNI, no significant difference was observed between PPS and non-PPS patients. This result indicated that the fat tissue thickness in the anterior mediastinum is not correlated 
to nutritional status and the physical constitution of the patients. Moreover, the decrement of body weight after surgery also did not correlated to the occurrence of PPS. The fat tissue in front of the mediastinum could be an anchor that can help maintain the normal position of the mediastinum after pneumonectomy. These findings suggested that the change of respiratory function and body weight after pneumonectomy could not be predictive factors and the diet after surgery to increase body weight might not be effect to prevent PPS.

In terms of respiratory function before surgery, the $\mathrm{VC}$ of PPS patients was higher than the median of non-PPS patients. The FEV1 was similar between PPS and non-PPS patients. By contrast, in the ppo-VC, the PPS-1 case was higher and the PPS-2 case was lower than the median of non-PPS cases. Some studies showed that hyperinflation of the residual lung was a factors of PPS $(1,2)$. However, the fat tissue between the bilateral thoracic space is a partition that can prevent the residual lung to hyperinflate toward the thoracic cavity after pneumonectomy.

Regarding to the affected side, PPS occurs more commonly after the right-sided pneumonectomy than the left-sided pneumonectomy in several reports $(1,3,12)$. Shen et al. reported 18 PPS patients, which developed after right pneumonectomy in 13 patients and after left pneumonectomy in 5 patients. Grillo et al. reported PPS, which followed right pneumonectomy in 7 patients and left pneumonectomy in 4 patients. Mehran et al. described that most cases of PPS have occurred after right pneumonectomy, because the powerful negative pressure of the involved hemothorax and the overexpansion of the remaining lung create a vacuum, moving the mediastinum toward the right side. According to these literatures, the occurrence of the left-sided PPS may be less than rightsided PPS. However, the left-sided pneumonectomy might be performed more often than right-sided pneumonectomy because right-sided pneumonectomy is often avoided in respiratory function.

The fat tissue thickness between the bilateral lungs can be a predictive factor for occurrence of PPS before surgery. By contrast, the changes of respiratory function and body weight after surgery did not differ in the two groups. To find the occurrence of PPS as soon as possible after surgery, the shift of the anterior mediastinum should be observed in the patients with the thin fat tissue in the anterior mediastinum before pneumonectomy.

The limitation of this study is small size retrospective cohort in one single institution, because pneumonectomy is becoming rarer due to several efforts made to reduce the numbers of pneumonectomy for the treatment of lung cancer. Therefore, it is difficult to collect the adequate patients undergone pneumonectomy. On the other hand, no similar observations to explore the predictive factors of PPS have been published, to our best knowledge. More studies with larger cohorts are needed in multi institutions, because the frequency of the pneumonectomy for lung cancer is decreasing due to the development of the other treatments.

\section{Conclusions}

In conclusion, the fat tissue in the anterior mediastinum between the bilateral lungs is an anchor that can help maintain the normal position of the mediastinum after pneumonectomy. Thus, the fat tissue thickness between the bilateral lungs can be a predictive factor for occurrence of PPS before surgery.

\section{Acknowledgments}

Funding: None.

\section{Footnote}

Reporting Checklist: The authors have completed the STROBE reporting checklist. Available at http://dx.doi. org/10.21037/jtd-20-2370

Data Sharing Statement: Available at http://dx.doi. org/10.21037/jtd-20-2370

Peer Review File: Available at http://dx.doi.org/10.21037/jtd20-2370

Conflicts of Interest: All authors have completed the ICMJE uniform disclosure form (available at http://dx.doi. org/10.21037/jtd-20-2370). The authors have no conflicts of interest to declare.

Etbical Statement: The authors are accountable for all 
aspects of the work in ensuring that questions related to the accuracy or integrity of any part of the work are appropriately investigated and resolved. The study was conducted in accordance with the Declaration of Helsinki (as revised in 2013). The study was approved by our institutional review board (Approval number, R2-3). Because of the retrospective nature of the study, patient informed consent or ethical statement was not required.

Open Access Statement: This is an Open Access article distributed in accordance with the Creative Commons Attribution-NonCommercial-NoDerivs 4.0 International License (CC BY-NC-ND 4.0), which permits the noncommercial replication and distribution of the article with the strict proviso that no changes or edits are made and the original work is properly cited (including links to both the formal publication through the relevant DOI and the license). See: https://creativecommons.org/licenses/by-nc-nd/4.0/.

\section{References}

1. Mehran RJ, Deslauriers J. Late complications. Postpneumonectomy syndrome. Chest Surg Clin N Am 1999;9:655-73, x.

2. Valji AM, Maziak DE, Shamji FM, et al. Postpneumonectomy Syndrome: Recognition and Management. Chest 1998;114:1766-9.

3. Shen KR, Wain JC, Wright CD, et al. Postpneumonectomy syndrome: surgical management and long-term results. J Thorac Cardiovasc Surg 2008;135:1210-6; discussion 1216-9.

4. Macaré van Maurik AFM, Stubenitsky BM, van Swieten HA, et al. Use of tissue expanders in adult

Cite this article as: Sugiura Y, Nakayama T, Hashizume T. Postpneumonectomy syndrome related to the thickness of the fat tissue in the anterior mediastinum: a retrospective observational study. J Thorac Dis 2020;12(11):6761-6768. doi: $10.21037 /$ jtd-20-2370 postpneumonectomy syndrome. The Journal of Thoracic and Cardiovascular Surgery 2007;134:608-12.

5. Uyama T, Monden Y, Sakiyama S, et al. Management of postpneumonectomy syndrome by intrapleural injection of sulfur hexafluoride. Case report. Scand J Thorac Cardiovasc Surg 1993;27:179-81.

6. Abe J, Hasumi T, Tanaka R, et al. Long-term outcome of nitinol stenting to treat asphyxia caused by postpneumonectomy syndrome. Respirol Case Rep 2017;5:e00207.

7. Cordova FC, Travaline JM, O'Brien GM, et al. Treatment of left pneumonectomy syndrome with an expandable endobronchial prosthesis. Chest 1996;109:567-70.

8. Gonzalez Madrono A, Mancha A, Rodriguez FJ, et al. The use of biochemical and immunological parameters in nutritional screening and assessment. Nutr Hosp 2011;26:594-601.

9. Huhmann MB, Cunningham RS. Importance of nutritional screening in treatment of cancer-related weight loss. Lancet Oncol 2005;6:334-43.

10. Buzby GP, Mullen JL, Matthews DC, et al. Prognostic nutritional index in gastrointestinal surgery. The American Journal of Surgery 1980;139:160-7.

11. Brunelli A, Kim AW, Berger KI, et al. Physiologic Evaluation of the Patient With Lung Cancer Being Considered for Resectional Surgery: Diagnosis and Management of Lung Cancer, 3rd ed: American College of Chest Physicians Evidence-Based Clinical Practice Guidelines. CHEST 2013;143:e166S-90S.

12. Grillo HC, Shepard JA, Mathisen DJ, et al. Postpneumonectomy syndrome: diagnosis, management, and results. Ann Thorac Surg 1992;54:638-50; discussion 650-1. 